\title{
Whole grain intake, incident hip fracture and presumed frailty in the Iowa Women's Health Study
}

\author{
David R. Jacobs $\mathrm{Jr}^{1,2}$, Christina Hohe ${ }^{3}$, Jaakko Mursu ${ }^{1}$, Kim Robien ${ }^{1}$ and Aaron R. Folsom ${ }^{1}$ \\ ${ }^{1}$ Division of Epidemiology and Community Health, School of Public Health, University of Minnesota, 1300 South 2nd Street, \\ Suite 300, Minneapolis, MN 55454, USA \\ ${ }^{2}$ Department of Nutrition, University of Oslo, Oslo, Norway \\ ${ }^{3}$ Department of Epidemiology, School of Public Health, University of California at Los Angeles, Los Angeles, CA, USA
}

(Received 10 February 2010 - Revised 23 April 2010 - Accepted 17 May 2010 - First published online 23 June 2010)

Whole cereal grain foods are rich in phytate, a Ca chelator, and could increase the risk of hip fracture. The objective of the present study was to investigate the association between baseline whole grain intake and incident hip fracture. In the present study, 29192 women who at baseline in 1986 were aged 55-69 years, free of diabetes, and reported a plausible energy intake of $2508-20900 \mathrm{~kJ} / \mathrm{d}$ and reported no fracture since the age of 35 years were followed. Hip fracture ( $n$ 746) was self-reported in five questionnaires through 2004. Of 1451 hip fractures identified passively by Medicare linkage through 31 December 2004 (Medicare hip fracture), 507 had also been self-reported. Whole grain intake was inversely related to Medicare hip fracture $\left(P_{\text {trend }}=0.02\right)$, but it was unrelated to self-reported hip fracture $\left(P_{\text {trend }}=0.27\right)$. The hazard ratio in the highest to lowest quintile of whole grain intake for incident Medicare-only hip fracture ( $n$ 944) was 0.66 (95\% CI 0.53, 0.82) after adjustment for age, energy intake, education, BMI, waist-to-hip ratio, farm residence, physical activity, oestrogen use, smoking, alcohol use, history of cancer and other dietary variables. Medicare-only cases may have failed to self-report due to severe illness; hazard ratio for total mortality after hip fracture was $2.92(2.37,3.59)$ for Medicare-only cases $v$. Medicare-confirmed self-reported cases. In conclusion, in this cohort, the inverse association between whole grain intake and hip fracture was explained by ascertainment bias. Whole grain intake may increase the ability to respond to a questionnaire and self-report hip fracture, and could reflect less undocumented frailty.

Bone: Epidemiological cohort studies: Statistical bias: Food-frequency questionnaires: Medicare linkage

Whole cereal grain consumption is thought to have health benefits such as protection against CHD and diabetes ${ }^{(1-7)}$. Accordingly, the 2005 US Dietary Guidelines recommend that at least half of the recommended 6-11 grain servings/d comprise whole grain foods ${ }^{(8)}$. However, one potential concern is whether the phytic acid in the whole grain foods leads to an increased risk of hip fracture by reducing the bioavailability of dietary minerals ${ }^{(9-16)}$, in particular, that of co-ingested $\mathrm{Ca}$. Thus, increased whole grain consumption could have an adverse effect on bone metabolism ${ }^{(13)}$, potentially increasing the risk of fracture ${ }^{(14)}$.

Most studies concerned with this phenomenon have been either in vitro ${ }^{(11)}$ or short-term human feeding studies which measured $\mathrm{Ca}$ binding or biomarkers of bone turnover ${ }^{(15,16)}$. Findings from balance studies ${ }^{(10,16)}$ have been inconsistent, possibly due to imprecise measurements of $\mathrm{Ca}$ absorption. Human studies ${ }^{(13,14,16)}$ have suggested a lack of association between high phytate/Ca diets or wheat bran fibre and markers of bone turnover or density; however, these studies have been limited by the small sample size, short duration of the intervention and other study design limitations.

In the present study, we evaluated the association between whole grain intake and risk of incident hip fracture in a large, prospective cohort of women, most of whom were postmenopausal. Based on the literature available to date, we hypothesised that there is no effect of whole grain or phytic acid intake on incident hip fracture risk. We actually observed an inverse association with hip fracture that was not self-reported. We therefore explored the characteristics of women who did not report their hip fracture, and considered the possibility that those hip fractures might be associated with a frail condition.

\section{Subjects and methods}

\section{Subjects}

The Iowa Women's Health Study was originally designed to study dietary and lifestyle factors and cancer incidence. In 1986, 99826 Iowa women aged 55-69 years were randomly selected from the 1985 Iowa Department of Transportation driver's license list and were sent a questionnaire; 41836 women (42\%) participated in the study as described previously ${ }^{(17)}$. On average, non-responders had a $0.4 \mathrm{~kg} / \mathrm{m}^{2}$ higher mean BMI, were 3 months younger, were more likely to live in rural counties and had a slightly lower 
mean income than responders ${ }^{(17)}$. The present analysis was carried out with 29192 women from the participating Iowa women's cohort ( $n$ 41 836). We followed the women for self-reported hip fracture using five questionnaires. We excluded women whose baseline questionnaire indicated selfreported diabetes ( $n$ 3007), energy intake outside a reasonable range $(<2508$ and $>20900 \mathrm{KJ} / \mathrm{d})(n$ 3102), a self-reported fracture between the age of 35 years and baseline ( $n$ 5863) or had missing data on variables used in the multivariable-adjusted model of the analysis ( $n$ 916); exclusions were not mutually exclusive. We additionally linked the women to the Centers for Medicare and Medicaid Services (CMS) database (Medicare records) to identify hip fractures that had not been self-reported. Some women had no CMS record, so the final sample size for the outcome variable hip fracture identified by CMS linkage was 28706 . About $98 \%$ of women were ever enrolled in Medicare.

The Iowa Women's Health Study was approved by the University of Minnesota Institutional Review Board, and return of the questionnaire was considered informed consent.

\section{Data collection}

The 1986 baseline questionnaire included questions on variables possibly relevant to hip fracture (demographic: age, weight, height, farm residence and educational level; lifestyle factors: smoking, alcohol consumption, hormone replacement therapy and physical activity; and medical conditions: cancer other than skin cancer). The first physical activity question queried whether any regular activity to keep fit was done. The two subsequent questions queried about the frequency of participation (rarely or never, a few times a year, a few times a month, about once a week, 2-4 times a week and more than 4 times a week) in moderate physical activities (e.g. bowling, golf, light sports or physical exercise, gardening and taking long walks) and vigorous physical activities (e.g. jogging, racquet sports, swimming, aerobics and strenuous sports). These questions were combined in a threelevel frequency score as less than a few times a month, a few times a month or once a week and two or more times a week. A tape measure was included with the questionnaire; waist and hip measurements were obtained by following the instructions and with the help of a friend, and waist-to-hip ratio was computed. A 127-item semi-quantitative FFQ was also included, which was adapted from the Nurses' Health Study, developed and validated within a subgroup of nurses aged 34-59 years of the Boston area ${ }^{(18)}$ and tested for reliability in 1988 within a subset of the present study's cohort of forty-four women giving several $24 \mathrm{~h}$ recalls ${ }^{(19)}$. The women indicated their average dietary intake in servings per week over the preceding year from nine categories (never or less than once per month, 1-3 per month, 1 per week, 2-4 per week, 1 per day, 2-3 per day, $4-5$ per day and $6+$ per day) for the listed food items, disregarding seasonal variations in the intake of fruits and vegetables.

The composite measure of whole grain intake (servings per week) has been well documented previously, and was the sum of servings per week consumed for dark bread, cold breakfast cereal, brown rice, popcorn, wheat germ, bran, cooked oatmeal and other grains (i.e. bulgar, kasha and couscous) ${ }^{(20)}$. Furthermore, the women were asked for the brand of their most commonly eaten breakfast cereal, which was counted as being whole grain if $\geq 25 \%$ of its weight was from whole grain or bran ${ }^{(21)}$. Energy intake, phytic acid and dietary Ca were calculated using the Harvard Nutrient Database ${ }^{(18)}$.

Five mailed follow-up questionnaires were collected in 1987, 1989, 1992, 1997 and 2004, with response rates of 91, $89,83,79$ and $69 \%$. Participants were queried whether they had suffered a fracture (broken bone) which required treatment by a doctor since the last survey date, and if so, whether it was a hip fracture. For hip fractures reported in the 1987 and 1989 follow-up questionnaires, detailed validation questionnaires were sent by Munger et al. ${ }^{(21)}$. Of the seventy-five detailed questionnaires returned, sixty-six $(88.0 \%)$ confirmed their original brief report of hip fracture, with errors being attributed to incorrect marking of the follow-up questionnaire or mistaking sprains or other pains for fractures. The hip fracture diagnosis was confirmed in all the forty-four cases in which the physician responded to a separate inquiry.

In the present study, hip fracture was self-reported by 746 women. CMS data linkage identified 1451 hip fractures (all acute care hospitalisations from 1986-2004 with an International Classification of Diseases 9 code of 820.XX in any of the ten discharge diagnosis fields). Of the 746 self-reported hip fractures, 507 also had a CMS report. No CMS hip fracture was found in 233 self-reported cases, 6 self-reported cases had no CMS record, and 944 hip fractures were found by CMS linkage only. Among the hip fractures that occurred at the age of at least 65 years (the age of Medicare eligibility for most people), 1379 were identified by CMS linkage and 662 were self-reported, of which, 496 also had a CMS report. Unconfirmed self-reported hip fracture may be partly errant reporting. If $88 \%$ of self-reports are correct ${ }^{(21)}$, about 90 of 746 self-reports would not actually be hip fractures, and these ninety would constitute $38 \%$ of the 239 selfreported cases that were not confirmed by CMS linkage. Unconfirmed self-report could also be attributed to age ineligibility for Medicare or treatment not captured by CMS (in a Health Maintenance Organisation or outside of Medicare).

For a self-reported hip fracture, total person-years at risk was computed from the time of the baseline questionnaire in 1986 until (a) the midpoint between the dates of the followup questionnaire first reporting a hip fracture and the questionnaire preceding it, (b) the date of death through 31 December 2004 or (c) the date of the last survey that was completed before 31 December 2004. For a CMS-identified hip fracture, total person-years at risk was computed similarly, except that the start of the follow-up was the date of the first Medicare enrolment and the end was the date of death or the first Medicare disenrolment. Deaths were identified through the Iowa State Health Registry and the National Death Index.

\section{Statistical analyses}

All data analyses were carried out with Statistical Analysis Systems software (version 9.2; SAS Institute, Inc., Cary, NC, USA). Proportional hazards regression analysis was used to compare hip fracture risk across quintiles of whole grain intake, with the lowest intake as the reference category. The $P$-value for risk trend was computed from a parallel regression analysis, in which whole grain was used as a continuous variable. Covariates in the fully adjusted model 
were age (continuous), energy intake (continuous), education, $\mathrm{BMI}, \mathrm{BMI}^{2}$, waist-to-hip ratio, farm residence (yes/no), physical activity (low, moderate and high), hormone replacement therapy (yes and no/not currently), smoking (currently, past and never), alcohol (g/d), total dairy (servings/ week), legumes (servings/week), fish and seafood (servings/ week), fruits (servings/week), vegetables (servings/week) and history of cancer at baseline (yes/no). Relative hazards with their respective $95 \% \mathrm{CI}$ are presented. Parallel analyses were carried out with phytic acid or the ratio of phytic acid to $\mathrm{Ca}$ intake as the predictor variable.

\section{Results}

As reported previously ${ }^{(3,6,22,23)}$, compared with women who rarely ate whole grain foods, habitual whole grain food consumers had an otherwise healthy lifestyle, including lower BMI, lower refined grain intake, higher fruit and vegetable intake, higher education, less smoking, and more oestrogen and multivitamin use, and were more likely to live on a farm (Table 1). As hypothesised, we found no significant difference in the risk of incident self-reported hip fracture across quintiles of whole cereal grain intake among the 29192 Iowa women, whether in the minimally adjusted or the multivariable-adjusted model (Table 2). In contrast, and contrary to our hypothesis, the risk of incident hip fracture identified by CMS decreased across quintiles of whole cereal grain intake among the 28706 Iowa women ever enrolled in Medicare, in both the minimally adjusted $\left(P_{\text {trend }}=0.003\right)$ and the multivariableadjusted $\left(P_{\text {trend }}=0 \cdot 02\right.$, Table 2$)$ models. The difference in the findings between the analyses of the two case ascertainment sources was more apparent in the analysis of the risk of incident hip fracture identified only by CMS, which was strong across quintiles of whole cereal grain intake $\left(P_{\text {trend }}<0.001\right.$, Table 2). The lack of association of whole grain intake with self-reported hip fracture (Table 2) was also found in the self-reported cases that were confirmed by CMS linkage (Table 2).

Additional analyses focused on the influence of case ascertainment method. First, polychotomous logistic regression in the multivariable-adjusted model found that the prediction coefficients for whole grain intake differed significantly for self-reported cases $v$. CMS-only cases $(P=0.0003)$. Secondly, within those who self-reported hip fracture $(n 746)$ or

Table 1. Hip fracture outcome variables and baseline characteristics across quintiles (Q) of whole grain intake (servings/week) among women aged 55-69 years at baseline, lowa Women's Health Study, 1986-2004

(Number of cases and percentage values or means)

\begin{tabular}{|c|c|c|c|c|c|c|c|c|c|c|c|}
\hline & \multicolumn{10}{|c|}{ Quintiles of whole grain food intake } & \multirow[b]{3}{*}{$P$ for trend } \\
\hline & \multicolumn{2}{|c|}{ Q1 (0-3.5) } & \multicolumn{2}{|c|}{ Q2 (4-7) } & \multicolumn{2}{|c|}{ Q3 (7.5-10.5) } & \multicolumn{2}{|c|}{ Q4 (10.8-18.5) } & \multicolumn{2}{|c|}{ Q5 $(19+)$} & \\
\hline & $n$ & $\%$ & $n$ & $\%$ & $n$ & $\%$ & $n$ & $\%$ & $n$ & $\%$ & \\
\hline Whole grain (servings/week) & \multicolumn{2}{|c|}{$1 \cdot 8$} & \multicolumn{2}{|c|}{$5 \cdot 6$} & \multicolumn{2}{|c|}{$8 \cdot 8$} & \multicolumn{2}{|c|}{14.5} & \multicolumn{2}{|c|}{25.7} & \\
\hline Hip fracture & & & & & & & & & & & \\
\hline Self-reported ( $n 746)$ & 131 & $2 \cdot 4$ & 143 & 2.5 & 152 & $2 \cdot 5$ & 168 & $2 \cdot 7$ & 152 & $2 \cdot 7$ & 0.81 \\
\hline Medicare linkage ( $n$ 1451) & 295 & 5.5 & 279 & $5 \cdot 0$ & 308 & $5 \cdot 2$ & 307 & $5 \cdot 1$ & 262 & $4 \cdot 6$ & 0.31 \\
\hline Medicare linkage only ( $n$ 944) & 209 & 3.9 & 182 & 3.2 & 208 & 3.5 & 194 & $3 \cdot 2$ & 151 & $2 \cdot 7$ & 0.007 \\
\hline Self-report and Medicare linkage ( $n$ 507) & 86 & 1.6 & 97 & 1.7 & 100 & 1.7 & 113 & 1.9 & 111 & $2 \cdot 0$ & 0.62 \\
\hline \multicolumn{12}{|l|}{ Baseline characteristics } \\
\hline Age (years) & $61 \cdot 1$ & $4 \cdot 2$ & $61 \cdot 0$ & $4 \cdot 2$ & $61 \cdot 4$ & $4 \cdot 2$ & $61 \cdot 5$ & $4 \cdot 2$ & $61 \cdot 5$ & $4 \cdot 2$ & $<0.0001$ \\
\hline BMI $\left(\mathrm{kg} / \mathrm{m}^{2}\right)$ & $27 \cdot 1$ & $5 \cdot 2$ & $27 \cdot 0$ & $5 \cdot 0$ & $26 \cdot 8$ & 4.8 & $26 \cdot 5$ & $4 \cdot 7$ & $26 \cdot 6$ & 4.9 & $<0.0001$ \\
\hline Waist-to-hip ratio & 0.84 & 0.09 & 0.83 & 0.09 & 0.83 & 0.08 & 0.83 & 0.08 & 0.83 & 0.08 & $<0.0001$ \\
\hline Smoking status (\%) & & & & & & & & & & & $<0.0001$ \\
\hline Currently & \multicolumn{2}{|c|}{$24 \cdot 4$} & \multicolumn{2}{|c|}{$16 \cdot 9$} & \multicolumn{2}{|c|}{$12 \cdot 1$} & \multicolumn{2}{|c|}{$10 \cdot 6$} & \multicolumn{2}{|c|}{$11 \cdot 3$} & \\
\hline Past & \multicolumn{2}{|c|}{$19 \cdot 2$} & \multicolumn{2}{|c|}{$20 \cdot 3$} & \multicolumn{2}{|c|}{$19 \cdot 4$} & \multicolumn{2}{|c|}{$19 \cdot 4$} & \multicolumn{2}{|c|}{$20 \cdot 8$} & \\
\hline Never & \multicolumn{2}{|c|}{$56 \cdot 5$} & \multicolumn{2}{|c|}{62.9} & 68 & & 70 & & 67 & & \\
\hline Education (\%) & & & & & & & & & & & $<0.0001$ \\
\hline $1-8$ years & 9 & & 7 . & & 7 . & & 6 . & & 6 . & & \\
\hline $9-12$ years & 12 & & 10 & & 8. & & 8. & & 8. & & \\
\hline High school graduate & 45 & & 43 & & 42 & & 40 & & 39 & & \\
\hline Beyond high school & 32 & & 38 & & 42 & & 45 & & 45 & & \\
\hline Physical activity frequency (\%) & & & & & & & & & & & $<0.0001$ \\
\hline Less than a few times a month & 58 & & 49 & & 46 & & 41 & & 40 & & \\
\hline A few times a month or once a week & 23 & & 27 & & 29 & & 29 & & 29 & & \\
\hline Two or more times a week & 17 & & 23 & & 24 & & 29 & & 30 & & \\
\hline Live on a farm (\%) & 17 & & 19 & & 19 & & 21 & & 19 & & $<0.0001$ \\
\hline Oestrogen use (\%) & 9 . & & 11 & & 12 & & 12 & & 13 & & $<0.0001$ \\
\hline Dietary intake & & & & & & & & & & & \\
\hline Energy $(\mathrm{kJ} / \mathrm{d}(\mathrm{kcal} / \mathrm{d}))$ & 6719( & 606) & 6990 & 671) & 7363 & 780) & 7876 & 882) & 8663 & 070) & $<0.0001$ \\
\hline Alcohol intake $(\mathrm{g} / \mathrm{d})$ & 5 & & & & & & & & & & $<0.0001$ \\
\hline Fruits (servings/week) & 16 & & 18 & & 20 & & 22 & & 24 & & $<0.0001$ \\
\hline Vegetables (servings/week) & 23 & & 26 & & 28 & & 30 & & 32 & & $<0.0001$ \\
\hline Legumes (servings/week) & 2 & & & & & & & & & & $<0.0001$ \\
\hline Dairy (servings/week) & 15 & & 16 & & 17 & & 18 & & 19 & & $<0.0001$ \\
\hline Fish and seafood (servings/week) & 1. & & & & & & & & & & $<0.0001$ \\
\hline
\end{tabular}

* $P$-value obtained from $t$ tests for continuous variables or from $\chi^{2}$ test for categorical variables. 
Table 2. Adjusted risk for incident self-reported hip fractures across quintiles (Q) of whole grain intake (servings/week) among women aged 55-69 years at baseline, lowa Women's Health Study, 1986-2004

(Hazard ratios and 95\% confidence intervals)

\begin{tabular}{|c|c|c|c|c|c|c|c|c|c|c|c|c|}
\hline & & \multicolumn{10}{|c|}{ Quintiles of total whole grain intake } & \multirow[b]{3}{*}{$P$ for trend } \\
\hline & & \multicolumn{2}{|l|}{ Q1 } & \multicolumn{2}{|c|}{ Q2 } & \multicolumn{2}{|c|}{ Q3 } & \multicolumn{2}{|c|}{ Q4 } & \multicolumn{2}{|c|}{ Q5 } & \\
\hline & & Hazard ratio & $95 \% \mathrm{Cl}$ & Hazard ratio & $95 \% \mathrm{Cl}$ & Hazard ratio & $95 \% \mathrm{Cl}$ & Hazard ratio & $95 \% \mathrm{Cl}$ & Hazard ratio & $95 \% \mathrm{Cl}$ & \\
\hline Servings/week & & \multicolumn{2}{|c|}{$0-3.5$} & \multicolumn{2}{|c|}{$4-7$} & \multicolumn{2}{|c|}{$7.5-10.5$} & \multicolumn{2}{|c|}{$10 \cdot 84-18.5$} & \multicolumn{2}{|c|}{$19+$} & \\
\hline Mean (SD) & & $1.8(1$ & & $5 \cdot 6$ & & $8 \cdot 8$ & & 14.5 & & $25 \cdot 7$ & & \\
\hline \multicolumn{13}{|l|}{ Self-reported hip fracture } \\
\hline Number at risk & 29192 & 5477 & & \multicolumn{2}{|c|}{5740} & \multicolumn{2}{|c|}{6060} & \multicolumn{2}{|c|}{6171} & \multicolumn{2}{|c|}{5744} & \\
\hline Cases & 746 & 131 & & \multicolumn{2}{|c|}{143} & \multicolumn{2}{|c|}{152} & \multicolumn{2}{|c|}{168} & \multicolumn{2}{|c|}{152} & \\
\hline Person (years) & 407571 & 7295 & & \multicolumn{2}{|c|}{79467} & \multicolumn{2}{|c|}{85725} & \multicolumn{2}{|c|}{87271} & \multicolumn{2}{|c|}{82157} & \\
\hline Minimally adjusted* ${ }^{*}$ & & 1 & & 1.00 & $0.79,1.27$ & 0.95 & $0.75,1.20$ & 1.01 & $0.81,1.26$ & 0.99 & $0.78,1.26$ & 0.62 \\
\hline Multivariable adjusted $\dagger$ & & 1 & & 1.08 & $0.85,1.38$ & 1.04 & $0.82,1.32$ & $1 \cdot 12$ & $0.89,1.43$ & $1 \cdot 11$ & $0.86,1.42$ & 0.27 \\
\hline \multicolumn{13}{|c|}{ Hip fracture identified by Medicare linkage } \\
\hline Number at risk & 28706 & 5357 & & \multicolumn{2}{|c|}{5634} & \multicolumn{2}{|c|}{5968} & \multicolumn{2}{|c|}{6079} & 56 & & \\
\hline Cases & 1451 & 295 & & 27 & & 30 & & 30 & & 26 & & \\
\hline Person (years) & & 6870 & & 73 & & 79 & & 826 & & 771 & & \\
\hline Minimally adjusted ${ }^{*}$ & & 1 & & 0.74 & $0.62,0.87$ & 0.81 & $0.69,0.95$ & 0.86 & $0.73,1.00$ & 0.87 & $0.74,1.02$ & 0.003 \\
\hline Multivariable adjusted $\dagger$ & & 1 & & 0.93 & $0.79,1.09$ & 0.93 & $0.79,1.09$ & 0.88 & $0.74,1.04$ & 0.81 & $0.68,0.96$ & 0.02 \\
\hline Hip fracture identified only & y Medicar & linkage & & & & & & & & & & \\
\hline Number at risk & 28706 & 5357 & & 56 & & 59 & & 60 & & 56 & & \\
\hline Cases & 944 & 209 & & 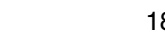 & & 20 & & 19 & & 15 & & \\
\hline Minimally adjusted ${ }^{*}$ & & 1 & & 0.79 & $0.65,0.97$ & 0.81 & $0.67,0.98$ & 0.71 & $0.58,0.86$ & 0.59 & $0.48,0.73$ & $<0.0001$ \\
\hline Multivariable adjusted $\dagger$ & & 1 & & 0.85 & $0.69,1.04$ & 0.89 & $0.73,1.08$ & 0.78 & $0.63,0.95$ & 0.66 & $0.53,0.82$ & $<0.001$ \\
\hline Hip fracture identified by $s$ & If-report ar & by Medicare lir & kage & & & & & & & & & \\
\hline Number at risk & 28706 & 5357 & & 56 & & 59 & & 60 & & 56 & & \\
\hline Cases & 507 & 86 & & 9 & & 10 & & 11 & & 11 & & \\
\hline Minimally adjusted* & & 1 & & 1.05 & $0.79,1.41$ & 0.97 & $0.73,1.30$ & 1.08 & $0.81,1.43$ & $1 \cdot 12$ & $0.84,1.50$ & 0.17 \\
\hline Multivariable adjusted $\dagger$ & & 1 & & $1 \cdot 12$ & $0.83,1.50$ & 1.03 & $0.77,1.39$ & $1 \cdot 13$ & $0.84,1.51$ & $1 \cdot 18$ & $0.87,1.59$ & 0.16 \\
\hline
\end{tabular}

${ }^{*}$ Adjusted for age (continuous) and energy intake (continuous).

† Adjusted for age (continuous), energy intake (continuous), education, BMI, BMI², waist-to-hip ratio, farm residence (yes/no), physical activity (low, moderate and high), hormone replacement therapy (yes and no/not currently), smoking (currently, past and never), alcohol (g/d), total dairy (servings/week), legumes (servings/week), fish and seafood (servings/week), fruits (servings/week), vegetables (servings/week) and cancer at baseline (yes/no). 
Table 3. Percentage of women who responded to the questionnaire in 2004 among 21770 women who were alive at the end of 2004

\begin{tabular}{|c|c|c|c|c|c|}
\hline & $\begin{array}{l}\text { Number of } \\
\text { women alive }\end{array}$ & $\begin{array}{l}\text { Number of women } \\
\text { responding }\end{array}$ & $\begin{array}{l}\text { Percentage } \\
\text { of women } \\
\text { responding (\%) }\end{array}$ & $z^{*}$ & $P$ \\
\hline Never had hip fracture & 21023 & 15242 & 73 & & \\
\hline $\begin{array}{l}\text { Self-report hip fracture through } 1997 \\
\text { (in the first four follow-up questionnaires) }\end{array}$ & 252 & 174 & 69 & $-1 \cdot 26$ & 0.21 \\
\hline $\begin{array}{l}\text { CMS-only hip fracture before the } \\
\text { end of } 1997\end{array}$ & 55 & 22 & 40 & $-4 \cdot 89$ & $<1 \times 10^{-06}$ \\
\hline $\begin{array}{l}\text { CMS-only hip fracture between } 1998 \\
\text { and } 2004\end{array}$ & 440 & 108 & 25 & -19.93 & $<1 \times 10^{-87}$ \\
\hline
\end{tabular}

CMS, Centers for Medicare and Medicaid Services.

* The $z$ statistic compares the proportion of women responding in the given row to those who never had a hip fracture, with corresponding two-sided $P$-value. Three hundred and twenty-one women who were alive at the end of 2004 and self-reported their first hip fracture in the 2004 questionnaire are omitted.

were CMS-only cases ( $n$ 944), $56 \%$ were CMS-only cases. In the multivariable-adjusted model using linear regression, CMS was the only source of ascertainment for $62 \%$ of the cases in the lowest whole grain intake quintile, which decreased progressively to $49 \%$ in the highest quintile.

Severe illness may have been involved in the failure to selfreport in the CMS-only cases. Self-reported hip fractures that were not reported by CMS occurred at an average age of 69.8 (SD 7.1) years compared with 74.8 (SD 5.2) years in selfreported cases that were also identified by CMS and $76 \cdot 3$ (SD 7.2) years in cases that were identified only by CMS. Death was much more frequent after a CMS-only hip fracture than after CMS-confirmed self-reported hip fracture (23 v. $48 \%$ through the end of the follow-up). The hazard ratio for total mortality after hip fracture was $2.92(2.37,3.59)$ for CMS-only cases $v$. those that were also self-reported, with multivariable adjustment as in Table 2. Furthermore, those who were alive at the end of 2004 with CMS-only hip fracture were by far the least likely to have responded to the questionnaire in 2004 (Table 3).

To evaluate whether the ascertainment source had a similar influence on another hip fracture risk factor, we assessed the relationship between baseline frequency of physical activity and risk of future hip fracture. In these analyses, physical activity frequency was inversely associated with the future hip fracture, and, in contrast to whole grain intake, the association was similar across different ascertainment sources. In the multivariable-adjusted proportional hazards regression analyses, those in the highest category of physical activity frequency had a hazard ratio of $0.83(0.73,0.96)$ for all the hip fractures identified by CMS compared with those in the lowest frequency. This compared to $0.78(0.64,0.95)$ for self-reported, $0.85(0.72,1.01)$ for CMS-only and 0.80 (0.63, 1.01) for CMS and self-reported hip fractures. Those with less frequent physical activity had an intermediate risk independent of ascertainment source, for example, 0.88 (0.75, 1.02) for all the cases identified by CMS.

Proportional hazards regression models (not shown) substituting phytic acid intake (correlation with whole grain food intake, 0.64) were in agreement with the lack of association of whole grain intake with self-reported hip fracture. Phytic acid, like whole grain food, was inversely associated with CMS-identified hip fracture $(P=0 \cdot 04)$. Phytic acid lost significance in a model that also contained whole grain food intake, while whole grain food intake remained strongly inversely associated with CMS-identified hip fracture $(P=0.002)$ in this model. In contrast, the ratio of phytic acid to $\mathrm{Ca}$ intake, which had a correlation with whole grain food intake of $0 \cdot 20$, did not show a significant association with incident hip fracture, whether self-reported or identified by CMS only.

\section{Discussion}

During 18 years of follow-up of women aged 55-69 years, whole grain food intake showed an inverse relationship with incident CMS hip fracture ( $n$ 1451, $P_{\text {trend }}=0.02$ ), but it was unrelated to self-reported incident hip fracture (n 746, $\left.P_{\text {trend }}=0 \cdot 27\right)$. However, we did observe a strong inverse association between whole grain food intake and incident hip fracture identified only through CMS linkage. It is unlikely that whole grain intake is causally related to the risk of hip fracture, since it is unrelated in a substantial subset of 507 CMS-confirmed, self-reported hip fractures. Therefore, our interpretation is that the overall inverse association is the result of ascertainment bias specifically related to whole grain intake. The CMS-only hip fractures were associated with severe illness, as illustrated by accelerated death rate and failure to respond to the 2004 questionnaire. In contrast, the amount and level of physical activity, a known risk factor for hip fracture ${ }^{(24)}$, were inversely associated with the risk of hip fracture, showing a similar pattern of risk reduction across all ascertainment sources. Thus, it is reasonable to speculate that many women whose hip fracture was identified only by CMS record linkage exhibited signs of frailty, although we do not have specific data to verify this assumption.

Self-reported hip fracture was partially validated in that it was generally identified in CMS records; however, more hip fractures were identified by CMS only than by self-report. In order to self-report a hip fracture, a questionnaire must be sent after the hip fracture had occurred and reach the subject, and the respondent must be in sufficiently good health to complete and return the questionnaire. Many CMS-only cases (women who did not self-report their hip fracture) may well have been incapacitated long after the event.

Although phytate in whole grains could have adverse effects on bone health, such effects were not apparent in the present study. A balance study found negative net $\mathrm{Ca}$ effects in subjects consuming a whole grain product ${ }^{(16)}$, but another study did not find such effects ${ }^{(10)}$. The inconclusive findings of balance studies have been attributed to imprecise measurements of $\mathrm{Ca}$ absorption ${ }^{(12)}$. Within a series of randomised 
crossover experiments, Weaver et al. ${ }^{(12)}$ created Ca-labelled wheat which allowed them to measure $\mathrm{Ca}$ absorption more precisely. They concluded that $\mathrm{Ca}$ absorption from wheat products was similar to that from milk, although phytaterich bran does interfere with co-ingested $\mathrm{Ca}$ absorption ${ }^{(12)}$.

In a study of nineteen healthy young women (mean age, 25.7 (SD 3.9) years), Zittermann et al. ${ }^{(16)}$ found no difference in serum biomarkers of bone turnover between the high-wheat bran fibre $(15 \mathrm{~g} / \mathrm{d})$ and low-wheat bran fibre $(1 \mathrm{~g} / \mathrm{d})$ supplementation groups. Similarly, in a study of healthy individuals in southern India, Harinarayan et al. ${ }^{(13)}$ found no difference in parathyroid hormone concentrations between 205 participants living in rural areas consuming high-phytate/Ca diets and 943 participants living in urban areas consuming low-phytate/Ca diets, although both the groups had low dietary $\mathrm{Ca}$ intake and were vitamin $\mathrm{D}$ deficient. Whole grain foods provide benefits to the consumers beyond the additive effects of their individual constituents ${ }^{(9)}$, which could explain why negative net $\mathrm{Ca}$ balance studies remain incongruent with serum biomarkers of bone turnover.

Our conclusion that there is no association between whole grain food intake and risk of future hip fracture is consistent with a randomised trial ${ }^{(14)}$. No adverse effect of 2-3 years of wheat bran supplementation (124 people assigned to eat $13.5 \mathrm{~g} / \mathrm{d}$ of wheat bran fibre supplement, delivered as loops, shredded cereal or bars, and 113 people assigned to eat $2 \mathrm{~g} / \mathrm{d}$ of the wheat bran fibre) on forearm bone mineral density was observed in a sample of men and women aged $40-80$ years.

Much of the beneficial effect of whole grain foods on other health outcomes has been attributed to their multifaceted constitution of dietary fibre, antioxidants, phytochemicals and other bioactive compounds (lignans, phytosterols, unsaturated fatty acids and anti-nutrients), which are thought to act together to provide protective health benefits to the consumers $^{(2)}$. Protease inhibitors, phytic acid, phenolics and saponins have been shown to reduce the risk of cancer of the colon and breast in animals. Phytic acid, lectins, phenolics, amylase inhibitors and saponins have also been shown to lower the plasma glucose, insulin and/or plasma cholesterol and TAG concentrations ${ }^{(9)}$. The synergistic effect of the individual constituents within the whole grains is yet to be explained, but it suggests that studies focusing on isolated constituents are to be interpreted with caution.

A limitation of the present study is its observational nature, because residual confounding is always a possibility. Although we have adjusted for lifestyle characteristics and aspects of diet other than whole grain food intake, there remains a possibility for the whole grain intake to be a marker for aspects of healthy lifestyle and diet that we did not measure. We were not able to exclude hip fracture due to causes other than osteoporosis, such as traumatic injuries. The present study is strictly generalisable only to older women. The strengths of the present study include the size and long follow-up.

We conclude that the inverse association of whole grain intake with hip fracture observed in the cohort that was studied reflects case ascertainment bias. It is of substantial methodological interest that correlates of self-reported hip fracture differ greatly from those of hip fracture identified by CMS, as has been observed in the Iowa Women's Health Study ${ }^{(25)}$. It is possible that whole grain intake has a role in limiting the risk of future frailty. However, because we did not specifically assess frailty, this possibility is stated as a hypothesis for future study.

\section{Acknowledgements}

The present study was supported by grant R01 CA39742 from the National Cancer Institute, Bethesda, MD, and a Fulbright Fellowship (Mursu). None of the authors has any conflict of interest. D. R. J. conceived the manuscript, performed data analysis, and wrote and critically reviewed the manuscript; C. H. and J. M. performed data analysis, and wrote and critically reviewed the manuscript; K. R. and A. R. F. critically reviewed the manuscript.

\section{References}

1. Jacobs DR \& Gallaher DD (2004) Whole grain intake and cardiovascular disease: a review. Curr Atheroscler Rep 6, 415-423.

2. Liu SM, Stampfer MJ, Hu FB, et al. (1999) Whole-grain consumption and risk of coronary heart disease: results from the Nurses' Health Study. Am J Clin Nutr 70, 412-419.

3. Jacobs DR, Meyer KA, Kushi LH, et al. (2009) Whole-grain intake may reduce the risk of ischemic heart disease death in postmenopausal women: the Iowa Women's Health Study. Am J Clin Nutr 68, 248-257.

4. Jensen MK, Koh-Banerjee P, Hu FB, et al. (2004) Intake of whole grains, bran and germ and risk of coronary heart disease among men. Am J Clin Nutr 80, 1492-1499.

5. Liu S, Buring JE, Sesso HD, et al. (2002) A prospective study of dietary fiber intake and risk of cardiovascular disease among women. J Am Coll Cardiol 39, 49-56.

6. Meyer KA, Kushi LH, Jacobs DR Jr, et al. (2000) Carbohydrates, dietary fiber, and incident type 2 diabetes in older women. Am J Clin Nutr 71, 921-930.

7. Montonen J, Knekt P, Jarvinen R, et al. (2003) Whole-grain and fiber intake and the incidence of type 2 diabetes. J Am Coll Nutr 77, 622-629.

8. US Department of Health and Human Services and US Department of Agriculture. U.S. Dietary Guidelines for Americans, 2005. Diet Guidelines Advisory Committee Report. Washington, DC: US Department of Health and Human Services, US Department of Agriculture. http://www.health.gov/DietaryGuidelines/ dga2005/report/default.htm (accessed 11 July 2008).

9. Slavin J (2003) Why whole grains are protective: biological mechanisms. Proc Nutr Soc 62, 129-134.

10. Wisker E, Nagael R, Tanudjaja TK, et al. (1991) Calcium, magnesium, zinc, and iron balances in young women: effects of a low-phytate barley-fiber concentrate. Am J Clin Nutr 54, $553-559$.

11. Siener R, Heynck H \& Hesse A (2001) Calcium-binding capacities of different brans under stimulated gastrointestinal $\mathrm{pH}$ conditions. In vitro study with ${ }^{45} \mathrm{Ca}$. J Agric Food Chem 49, 4397-4401.

12. Weaver CM, Heaney RP, Martin BR, et al. (1991) Human calcium absorption from whole-wheat products. J Nutr 121, 1769-1775.

13. Harinarayan C, Ramalakshmi T, Prasad UV, et al. (2007) High prevalence of low dietary calcium, high phytate consumption, and vitamin $\mathrm{D}$ deficiency in healthy south Indians. Am J Clin Nutr 85, 1062-1067.

14. Chen Z, Stini WA, Marshall JR, et al. (2004) Wheat bran fiber supplementation and bone loss among older people. Nutrition 20, 747-751. 
15. Knox TA, Kassarjian Z, Dawson-Hughes B, et al. (1991) Calcium absorption in elderly subjects on high- and lowfiber diets: effects of gastric acidity. Am J Clin Nutr 53, $1480-1486$.

16. Zittermann A, Scheld K, Danz A, et al. (1999) Wheat bran supplementation does not affect biochemical markers of bone turnover in young adult women with recommended calcium intake. Br J Nutr 82, 431-435.

17. Folsom AR, Kushi LH, Anderson KE, et al. (2000) Associations of general and abdominal obesity with multiple health outcomes in older women: the Iowa Women's Health Study. Arch Intern Med 160, 2117-2128.

18. Willett WC, Sampson L, Brown ML, et al. (1998) The use of a self-administered questionnaire to assess diet four years in the past. Am J Epidemiol 127, 1888-1899.

19. Munger RG, Folsom AR, Kushi LH, et al. (1992) Dietary assessment of older Iowa women with a food frequency questionnaire: nutrient intake, reproducibility, and comparison with 24-hour dietary recall interviews. Am J Epidemiol 136, 192-200.
20. Jacobs DR, Meyer KA, Kushi LH, et al. (1998) Whole grain intake may reduce the risk of ischemic heart disease death in postmenopausal women: the Iowa Women's Health Study. Am J Clin Nutr 68, 248-257.

21. Munger RG, Cerhan JR \& Chiu BC (1999) Prospective study of dietary protein and risk of hip fracture in postmenopausal women. Am J Clin Nutr 69, 147-152.

22. Jacobs DR, Anderson LF \& Blomhoff R (2007) Whole grain consumption with a reduced risk of noncardiovascular, noncancer death attributed to inflammatory diseases in the Iowa Women's Health Study. Am J Clin Nutr 85, 1606-1614.

23. Nicodemus KK, Folsom AR \& Anderson KE (2001) Menstrual history and risk of hip fractures in postmenopausal women: The Iowa Women's Health Study. Am J Epidemiol 153, 251-255.

24. Kohrt WM, Bloomfield SA, Little KD, et al. (2004) American College of Sports Medicine Position Stand: physical activity and bone health. Med Sci Sports Exerc 36, 1985-1996.

25. Virnig B, Durham S, Cerhan J, et al. (2010) Linking the Iowa Women's Health Study cohort to Medicare data: linkage results and application to hip fracture. Am J Epidemiol (In the Press). 